\title{
RESEARCH ARTICLE \\ Chickpea rhizobia symbiosis genes are highly conserved across multiple Mesorhizobium species
}

\author{
Marta Laranjo ${ }^{1,2}$, Ana Alexandre ${ }^{1,2}$, Raúl Rivas ${ }^{3}$, Encarna Velázquez ${ }^{3}$, J. Peter W. Young ${ }^{4}$ \& Solange \\ Oliveira ${ }^{1,2}$ \\ ${ }_{1}^{1}$ Departamento de Biologia, Universidade de Évora, Évora, Portugal; ${ }^{2}$ Laboratório de Microbiologia do \\ Solo - I.C.A.M. (Instituto de Ciências Agrárias Mediterrânicas), Universidade de Évora, Évora, Portugal; ${ }^{3}$ \\ Departamento de Microbiologia y Genetica, Universidad de Salamanca, Salamanca, Spain; and ${ }^{4}$ \\ Department of Biology, University of York, York, UK \\ Correspondence: Solange Oliveira, Departamento de Biologia, Universidade de Évora, Apartado 94, \\ 7002-554 Évora, Portugal. Tel.: +351 266 760878; fax: +351 266 760914; e-mail: ismo@uevora.pt
} Editor: Kornelia Smalla

\section{KEYWORDS}

rhizobia $\bullet$ chickpea $\bullet$ nifH $\bullet$ nodC $\bullet$ lateral gene transfer $\bullet$ symbiosis genes ABSTRACT

Chickpea has been considered as a restrictive host for nodulation by rhizobia. However, recent studies have reported that several Mesorhizobium species may effectively nodulate chickpea. With the purpose of investigating the evolutionary relationships between these different species with the ability of nodulating the same host, we analysed 21 Portuguese chickpea rhizobial isolates. Symbiosis genes nifH and nodC were sequenced and used for phylogenetic studies. Symbiotic effectiveness was determined to evaluate its relationship with symbiosis genes. The comparison of 16S rRNA gene-based phylogeny with the phylogenies based on symbiosis genes revealed evidence of lateral transfer of symbiosis genes across different species. Chickpea is confirmed as a nonpromiscuous host. Although chickpea is nodulated by many different species, they share common symbiosis genes, suggesting recognition of only a few Nod factors by chickpea. Our results suggest that sequencing of nifH or nodC genes can be used for rapid detection of chickpea mesorhizobia.

Received 19 February 2008; revised 26 May 2008; accepted 30 July 2008.

First published online 15 September 2008. 